\title{
UV-VIS SPECTRA AS POTENTIAL PROCESS ANALYTICAL TECHNOLOGY (PAT) FOR MEASURING THE DENSITY OF COMPRESSED MATERIALS: EVALUATION OF THE CIELAB COLOR SPACE
}

\author{
$\underline{\text { Tim D. Lillotte }^{1}, \text { Maike Joester }^{3} \text {, Benjamin Frindt }}{ }^{2}$, Andreas Berghaus ${ }^{3}$, Robert F. Lammens ${ }^{2}$, Karl G. Wagner ${ }^{1}$ \\ ${ }^{1}$ Department of Pharmaceutical Technology and Biopharmaceutics, University of Bonn, Gerhard-Domagk-Straße 3, \\ 53121 Bonn, Germany; karl.wagner@uni-bonn.de \\ ${ }^{2}$ Solids Development Consult GmbH, Peter-Joseph-Lenné-Str. 11, 51377 Leverkusen, Germany \\ ${ }^{3}$ ColVisTec AG, Max-Planck-Str. 3, 12489 Berlin-Adlershof, Germany
}

\begin{abstract}
In this study, a novel approach was developed for determining the density of compacts using ultraviolet-visible spectrophotometry. The assumption within this context was that a change in density affects the corresponding color information of the compact. From the obtained spectra of the visible range, the color information of the compact was calculated which turned out to be directly proportional to the density of the compact. In comparison, the obtained spectra were analyzed using partial least square regression. The results of this study showed that both methods could be used predicting the density of a compact from the corresponding visible spectrum at identical accuracy. In contrast to the partial least square regression, the correlation of the color information as a direct output parameter of the spectrophotometer with the density required no excessive data pre-processing. Subsequently, the easier and faster data processing of the color information over the partial least square regression, conceives using this novel approach as potential process analytical technology tool for implementation into a compression process e.g., tableting or roller compaction.
\end{abstract}

Keywords: process analytical technology (PAT), color space, tablet density, spectrophotometry, partial least square regression (PLS)

\section{INTRODUCTION}

The monitoring and controlling of the tablet or ribbon density and its corresponding porosity or solid fraction as critical quality attributes (CQAs) have become a major area of interest in the pharmaceutical industry with the goal of implementing fast and reliable measurement methods with the potential being used in process analytical technology (PAT) to ensure product quality particularly during continuous manufacturing. During the last decades, various methods have been investigated that are based on electromagnetic radiation (NIR, THz etc.). The corresponding electromagnetic spectra are used for the analysis of the density or other mechanical properties, which is usually performed by multivariate data analysis using principal component analysis (PCA) and partial least square (PLS) regression.

However, little has been reported about the use of visible (VIS) spectroscopy for the purpose of monitoring CQAs of pharmaceutical compacts. Therefore, the current study aims to investigate the influence of densification on the corresponding VIS spectra (380 to $780 \mathrm{~nm}$ ), with the goal to evaluate the use of this effect for determining the density of compacts. In this context, spectral data of different tablets were obtained with a UV-VIS spectrophotometer from ColVisTec AG (Berlin, Germany). In the present work, it was assumed that a change in the density of a compact affects the corresponding $\mathrm{L}^{*}, \mathrm{a}^{*}, \mathrm{~b}^{*}, \mathrm{C}^{*}$, and $\mathrm{h}^{\circ}$ values of the CIELAB color space. These values were determined for the compacts followed by comparing the calculated $\mathrm{C}^{*}$ values with the density values determined from the geometry and weight of the compact. The obtained regression functions were used as prediction models for the density, and the properties of these functions were compared to PLS regression results to assess the use of the CIELAB color space as a novel tool for predicting the density of compacts.

Note: This abstract only focuses on the evaluation of the CIELAB color space of one of seven blends. The results of the PLS regression and its comparison with the results of the CIELAB color space is featured in [1]. 


\section{MATERIAL AND METHODS}

A blend consisting of $99.5 \%$ Microcelac $\AA 100$ and $0.5 \%$ Ligamed $\AA$ was produced for the production of tablets. Six additional blends were made. However, the results are not shown in this abstract. Please refer to [1] for more information. A FlexiTab ${ }^{\circledR}$ (Röltgen $\mathrm{GmbH}$, Germany) was utilized to produce four tablet batches. Based on the geometry and the weight of the tablets, the respective tablet (envelope) density was calculated.

\section{ULTRAVIOLET-VISIBLE MEASURMENTS}

The tablets were investigated using a spectrophotometer "InSpectro X" (ColVisTec AG, Germany). The probe was attached to a $3 \mathrm{D}$ printed template holding the tablet under investigation in a defined position. Based on the raw spectral data, the device calculated the $L^{*}, a^{*}, b^{*}$, $\mathrm{C}^{*}$, and $\mathrm{h}^{\circ}$ values using the spectral information in the range of 380 to $780 \mathrm{~nm}$ for each measurement.

\section{CIELAB COLOR SPACE}

Linear regression was used to investigate whether the $\mathrm{C}^{*}$ value systematically correlated with the density of the tablet.

\section{RESULTS}

From the raw spectral data curves, the UV-VIS spectrophotometer calculated the $\mathrm{L}^{*}, \mathrm{a}^{*}, \mathrm{~b}^{*}, \mathrm{C}^{*}$, and $\mathrm{h}^{\circ}$ values of the sample under investigation. No preprocessing of the spectral data was performed. The $\mathrm{C}^{*}$ value was calculated based on the $a^{*}$ and $b^{*}$ values and was used for a direct correlation with the measured (envelope) density of each individual tablet, according to the assumption that a change in density affects the corresponding color information of the sample. The results of the regression analyses are shown in Fig. 1. For each blend, a good linear relation between the $\mathrm{C}^{*}$ value and the density of the tablets was observed, despite the overlap and shift in the raw spectra.

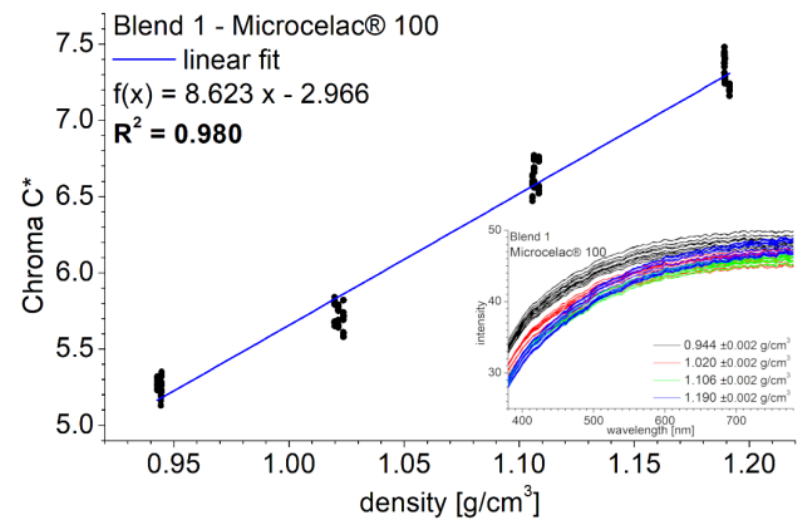

Fig. 1. Linear correlation of the $C^{*}$ value with the tablet (envelope) density.

\section{DISCUSSION}

The present study was designed to assess the applicability of the use of a compacts color information for determining its density. The assumption within this context was that a change in density affects the corresponding $\mathrm{C}^{*}$ value of the compact. The $\mathrm{C}^{*}$ value is a direct output parameter of the UV-VIS device and sufficiently sensitive to a change in density for predicting the density of compacts. The correlation of the $C^{*}$ value with the density can be easily and quickly performed and no additional software tools are required. The spectrophotometer is directly ready to use after calibration using polytetrafluoroethylene (Teflon $\left.{ }^{\circledR}\right)$. Moreover, no pre-processing of the raw spectral data sets is necessary and the operator can use well-known linear regression to correlate the color information with the density. Altogether, this substantially reduces the amount of work required to establish and to maintain a model for predicting the density and applying it in pharmaceutical processes. Additionally, no skills training in multivariate data analysis and no advanced spectroscopic knowledge is required. This is an advantage over other spectroscopic techniques such as NIR or THz.

\section{CONCLUSIONS}

The present study provides a novel method for measuring the density of pharmaceutical compacts, demonstrating that the $\mathrm{C}^{*}$ value can be used for this purpose. Please refer to [1] for the following:

The correlation of the $C^{*}$ value with the density was compared to the correlation based on PLS regression. The results of this comparison showed that the correlation results of the $\mathrm{C}^{*}$ value were just as accurate as the results by PLS regression. The results of this work support the idea that the color information of the sample can be easily and effectively used for measuring the density using VIS spectroscopy, without the use of timeconsuming and complex spectral analysis based on PCA and PLS regression.

\section{REFERENCES}

[1] T.D. Lillotte, M. Joester, B. Frindt, A. Berghaus, R.F. Lammens, K.G. Wagner, UV-VIS spectra as potential process analytical technology (PAT) for measuring the density of compressed materials: Evaluation of the CIELAB color space, International Journal of Pharmaceutics. $603 \quad$ (2021) 120668. https://doi.org/10.1016/j.ijpharm.2021.120668. 